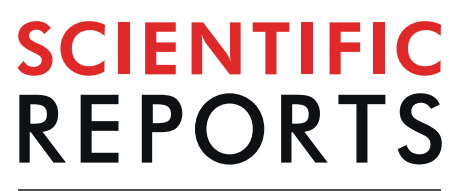

\title{
RBV: Read balance validator, a tool for prioritising copy number variations in germline conditions
}

\begin{abstract}
Whitney Whitford ${ }^{10}{ }^{1,2 *}$, Klaus Lehnert ${ }^{1,2}$, Russell G. Snell ${ }^{1,2}$ \& Jessie C. Jacobsen ${ }^{1,2}$
The popularisation and decreased cost of genome resequencing has resulted in an increased use in molecular diagnostics. While there are a number of established and high quality bioinfomatic tools for identifying small genetic variants including single nucleotide variants and indels, currently there is no established standard for the detection of copy number variants (CNVs) from sequence data. The requirement for CNV detection from high throughput sequencing has resulted in the development of a large number of software packages. These tools typically utilise the sequence data characteristics: read depth, split reads, read pairs, and assembly-based techniques. However, the additional source of information from read balance (defined as relative proportion of reads of each allele at each position) has been underutilised in the existing applications. Here we present Read Balance Validator (RBV), a bioinformatic tool that uses read balance for prioritisation and validation of putative CNVs. The software simultaneously interrogates nominated regions for the presence of deletions or multiplications, and can differentiate larger CNVs from diploid regions. Additionally, the utility of RBV to test for inheritance of CNVs is demonstrated in this report. RBV is a CNV validation and prioritisation bioinformatic tool for both genome and exome sequencing available as a python package from https:// github.com/whitneywhitford/RBV.
\end{abstract}

There are four main types of variation in the human genome: single nucleotide variants (SNVs), small-scale changes in genomic content in the form of short indels, structural variants, and aneuploidies. Structural variants consist of medium to large-scale changes to the genomic structure, and includes both balanced chromosomal rearrangements (such as inversions and translocations) and copy number variants (CNVs). CNVs are typically defined as deletions or multiplications of sections of the genome, resulting in changes of genomic content greater than $1 \mathrm{~kb}^{1}$. Initial efforts to map genetic variation on the whole genome scale indicated that SNVs constituted the majority of variation between individuals ${ }^{2}$. However, large scale collaborations mapping CNVs in the human genome found on average an individual harbours over $1,000 \mathrm{CNVs}$ of $443 \mathrm{bp}$ or greater ${ }^{3-6}$. Taken together, although there is a greater number of SNVs per individual (approximately 3.6 million or $\sim 0.1 \%$ of the genome ${ }^{5}$ ), due to the greater average size of CNVs and indels, they are responsible for greater genomic variance between genomes (up to $48.8 \mathrm{Mb}$ or $\sim 1.5 \%{ }^{6}$ ).

CNVs play an important role in gene expression with changes in genetic content larger than $1 \mathrm{Mb}$ estimated to be responsible for $17.7 \%$ of the genetic impact on gene expression ${ }^{7}$. One would expect that the proportion of genetically controlled variation in gene expression attributable to CNVs would be higher if CNVs smaller than $1 \mathrm{Mb}$ were included in such analyses. CNVs are able to affect gene expression directly through copy number changes of genes and regulatory elements ${ }^{8}$, and indirectly through unmasking of recessive alleles ${ }^{9}$ and positional effects $^{10}$. As such, there has been an increasing volume of research into the role of CNVs in disease. In particular, CNVs have been implicated in the aetiology of neuropsychiatric disorders including schizophrenia, intellectual disability, and autism spectrum disorder (as reviewed by Malhotra \& Sebat, 2012 $2^{11}$ ). Therefore, chromosomal microarray (CMA) has become a first-tier clinical diagnostic test for patients with unexplained intellectual disability, autism spectrum disorder, or multiple congenital anomalies, with diagnostic yield of 15-20\% (reviewed by Miller, et al. ${ }^{12}$ ). The use of high throughput sequencing (HTS) in the form of whole exome sequencing (WES) and whole genome sequencing (WGS) is increasing for diagnostic testing, both due to its decreasing cost and ability to investigate genetic variants without prior hypotheses. HTS based methods offer the potential of identifying SNVs, indels and CNVs (including those not detected by current diagnostic CMA thresholds ${ }^{13}$ ) in a single test. 

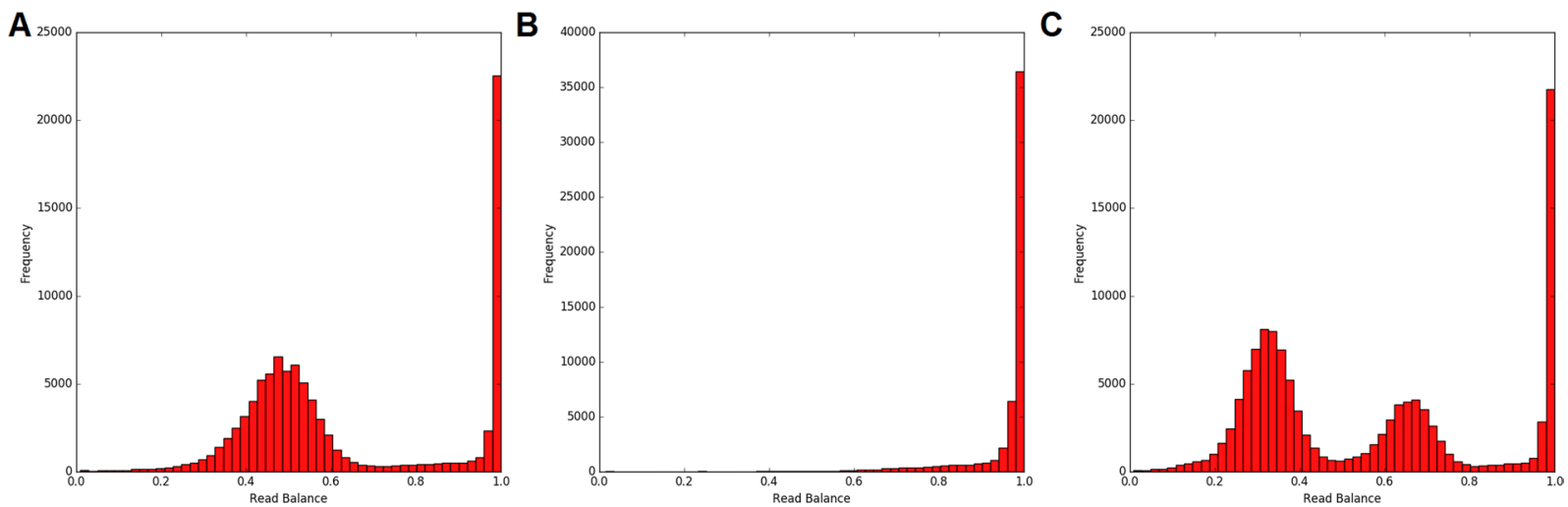

Figure 1. Distribution of relative reads for diploid, haploid, and triploid regions in whole genome sequence. (A) Expected distribution of all positions in a diploid genome. (B) Expected distribution of all positions in a hemizygous genome. (C) Expected distribution of all positions in a triploid genome.

With the rapid implementation of HTS in molecular diagnostics and research, there has been a proliferation of tools for variant identification. There are currently over 80 software packages designed to identify CNVs from WGS alone ${ }^{14}$. These tools predominantly rely on four characteristics of the sequence data: read depth, split reads, read pairs, and assembly-based techniques (reviewed by Zhao, et al. ${ }^{15}$ ). As yet underutilised, the allele balance of reads at a position contributes additional data that can also be exploited for $\mathrm{CNV}$ variant detection and validation. This 'read balance' is computed from relative read coverage of each allele at a given locus. The read balance can provide information regarding the copy number over the region in the form of the allele-specific copy number (ASCN). Positions in diploid regions of the genome are primarily invariant (homozygous) (as demonstrated in Fig. 1A). This is represented by a relative read distribution peak about 1 . The heterozygous positions (SNVs) are represented by a normal distribution centred on 0.5 , with the reads split evenly across the two alleles. A deleted (hemizygous or nullizygous) region should not contain any heterozygous positions; nullizygous regions by virtue of not containing genetic information for the aligned region, and hemizygous regions due to containing a single copy of the non-deleted allele, thus resulting in a distribution peak centred around 1, as depicted in Fig. 1B. A triplicated region as represented in Fig. 1C, however, is expected to have homozygous SNVs along with the heterozygous SNVs represented by two normal distributions centred on 0.33 and 0.66 , indicating that one third of the reads at a given locus include one allele, and two thirds of the reads include the other.

A number of bioinformatic tools have utilised ASCN for determining CNVs in cancer samples ${ }^{16-24}$. These techniques rely on sequence data from paired tumour and normal tissue samples, and therefore are not suitable for identifying germline CNVs. Alternatively, AS-GENSENG ${ }^{25}$ and ERDS ${ }^{26}$ incorporate read balance information into their algorithms along with read depth based data to discover CNVs. However, there is currently no independent platform providing validation of CNVs using read balance, allowing for integration of this additional data source in established bioinformatic pipelines that use alternative CNV discovery tools. RBV utilises read balance data to validate CNVs identified by other software packages, allowing for prioritisation of CNVs in both research and molecular diagnostic settings.

\section{Implementation}

RBV is a python package, which incorporates the read balance data from positions within the CNV of interest with randomly sampled windows across the genome to predict the authenticity of CNVs. The software extracts the read balance information from a variant call format (vcf) file, uses CNV coordinates from an interval list, and can be employed for both WGS and WES generated data. The analyses can be refined by restricting investigation to callable regions or outside of known gaps in the reference through the inclusion of either an interval list of callable regions, or an interval list of gaps in the reference genome provided by the user. The user can alter the specificity of RBV results through adjusting the parameters: quality and depth cut-offs at each position in the vcf, readbal cut-off for deletion analyses, and the number of randomly generated permutations for the positions and windows. RBV can incorporate data derived from popular variant callers (HaplotypeCaller ${ }^{27}$, SAMtools ${ }^{28}$, Freebayes $^{29}$, and Platypus ${ }^{30}$ ), and all aligners. However, issues with read balance calculations may arise from non-uniquely aligned regions of the genome if the aligner of choice places these reads at more than one position in the genome, or regions with non-uniform alignment. We therefore recommend using aligners that randomly place reads to only one mappable location by default, such as $\mathrm{BWA}^{31}$, and removing regions with low mappability and low sequence complexity by including an intervals file such as that provided in the GATK resources bundle ${ }^{27}$.

$\mathrm{RBV}$ is freely available via https://github.com/whitneywhitford/RBV.

\section{Results}

The analysis performed by RBV validates two separate hypotheses: that the putative CNV is a deletion with the region being hemizygous or nullizygous, or that the putative CNV is multiplicated where the region is triploid or greater. 

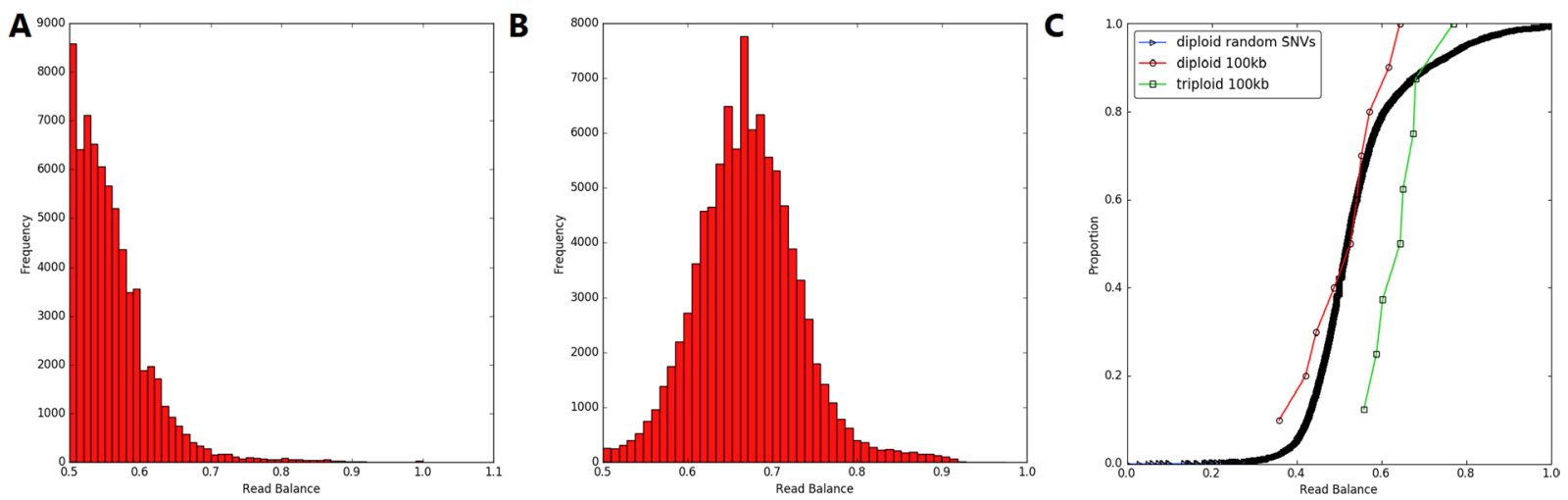

Figure 2. RBV data analysis curves. (A) Read balance of the most common allele from heterozygous positions in a diploid genome. (B) Read balance of the most common allele from heterozygous positions in a triploid genome. (C) CDF curve utilised in a 2-sample KS test, comparing distribution of read balance between randomly generated heterozygous SNVs throughout the reference diploid genome: a $100 \mathrm{~kb}$ diploid region, and a $100 \mathrm{~kb}$ triplicated region.

Deletion analyses. Deletions should represent areas of absence of heterozygosity (AOH), therefore the probability that a deletion exists (p-value) is calculated based on an empirical cumulative distribution function (eCDF). For this calculation, a large number of diploid windows (default 1,000) of the same number of callable base pairs as the CNV of interest are randomly generated from callable regions (if specified by the user) within the individual's genome, and the number of heterozygous SNVs in each window is subsequently calculated. The empirical p-value is calculated using the eCDF (Eq. 1) for the resulting distribution, with the probability being the proportion of randomly generated windows containing the same number or fewer heterozygous SNVs for the CNV in question.

$$
\text { Deletion p-value }=\frac{1}{n} \sum_{i=1}^{n} 1_{\mathrm{x}_{i} \leq t}
$$

where $x_{1}, \ldots, x_{n}$ represent the number of heterozygous SNVs within each randomly selected window in the eCDF equation where $n$ is the number of randomly generated windows of the same size as the CNV, and $t$ is the number of heterozygous SNVs within the CNV of interest.

Multiplication analyses. The multiplication hypothesis is interrogated using the two-sample KolmogorovSmirnov (KS) test. For this analysis we only consider the most common allele at each heterozygous position, which gives the distribution demonstrated in Fig. 2A,B. The differences in the distribution of read balance for randomly generated diploid heterozygous SNVs and the heterozygous SNVs (default 10,000) in the putative CNV are compared using the two-sample KS test, represented in Fig. 2C.

Performance. To analyse the performance of RBV, 25 high coverage whole genome alignments and their associated CNV calls $>1 \mathrm{~kb}$ from Phase 3 of the 1000 Genomes Project ${ }^{32}$ were downloaded. In order to facilitate comparison, diploid regions were randomly generated covering the same number of callable positions ${ }^{27}$ for each deletion and the same number of heterozygous SNVs for each duplication. For the 31,791 CNVs $(23,851$ deletions, and 7,940 duplications) analysed, RBV was able to identify statistically significant $(\mathrm{P} \geq 0.05) \mathrm{CNVs}$ with an overall sensitivity of $6.1 \%$ and $41.4 \%$ along with a specificity of $99.7 \%$ and $47.9 \%$, for deletions and duplications, respectively (Tables 1 and 2). The ability of RBV to prioritise CNVs over the randomly generated regions is demonstrated in Fig. 3.

The comparison shows a separation between CNVs and random regions, with an enrichment of CNVs with low p-values. The enrichment is increasingly marked for CNVs of greater size or greater number of heterozygous SNVs. Therefore, RBV has reduced sensitivity to detect smaller CNVs ( $<30 \mathrm{~kb}$ for deletions and $<20$ heterozygous SNVs for duplications) due to the reliance upon relatively infrequent heterozygous positions in the randomly generated windows for deletion analysis, and the increased power of a 2-sample KS test with a greater number of heterozygous positions in the CNV. These inherent biases are responsible for the poor overall sensitivity and specificity, and we recommend using RBV for prioritising deletions $>30 \mathrm{~kb}(82.1 \%$ sensitivity and $95.9 \%$ specificity) and duplications with at least 20 heterozygous SNVs (81.6\% sensitivity and $84.8 \%$ specificity).

In order to determine the performance of RBV based on the number of random windows sampled per deletion (used to calculate the eCDF), a power analysis was performed using a subset of six of the 1000 Genomes Phase 3 individuals from different populations, consisting of 1,358 deletions in total. RBV was run using 100, 500, 1000 (default), 5000 and 10000 random window permutations per deletion with analyses separated into six bin sizes (1-10 kb, 10-50 kb, 50-100 kb, 100-500 kb, $500 \mathrm{~kb}-1 \mathrm{Mb}$, and $1 \mathrm{Mb}+)$. Sensitivity and specificity $(\mathrm{P} \geq 0.05$ deletion vs. random diploid) was consistent for all bin sizes for random window permutations 500 and greater. The size of deletion had a far greater effect on the ability of RBV to sensitively and accurately identify deletions than random window permutations, where deletions $1-10 \mathrm{~kb}$ had a sensitivity of $0 \%, 10-50 \mathrm{~kb}$ had a sensitivity 


\begin{tabular}{|l|l|l|l|l|l|l|l|}
\hline Size & Total & TP & FN & TN & FP & Sensitivity & Specificity \\
\hline$>10 \mathrm{~kb}$ & 3783 & 1459 & 2324 & 3703 & 80 & 0.3856727 & 0.978853 \\
\hline$>20 \mathrm{~kb}$ & 1914 & 1254 & 660 & 1846 & 68 & 0.6551724 & 0.964472 \\
\hline$>30 \mathrm{~kb}$ & 1326 & 1089 & 237 & 1271 & 55 & 0.821267 & 0.958522 \\
\hline$>50 \mathrm{~kb}$ & 738 & 643 & 95 & 702 & 36 & 0.8712737 & 0.95122 \\
\hline$>100 \mathrm{~kb}$ & 397 & 374 & 23 & 375 & 22 & 0.9420655 & 0.944584 \\
\hline$>150 \mathrm{~kb}$ & 169 & 162 & 7 & 161 & 8 & 0.9585799 & 0.952663 \\
\hline$>200 \mathrm{~kb}$ & 93 & 88 & 5 & 89 & 4 & 0.9462366 & 0.956989 \\
\hline$>300 \mathrm{~kb}$ & 55 & 54 & 1 & 52 & 3 & 0.9818182 & 0.945455 \\
\hline$>400 \mathrm{~kb}$ & 31 & 31 & 0 & 30 & 1 & 1 & 0.967742 \\
\hline$>500 \mathrm{~kb}$ & 20 & 20 & 0 & 20 & 0 & 1 & 1 \\
\hline$>1 \mathrm{Mb}$ & 10 & 10 & 0 & 10 & 0 & 1 & 1 \\
\hline All & 23851 & 1459 & 22392 & 23771 & 80 & 0.0611714 & 0.996646 \\
\hline
\end{tabular}

Table 1. RBV performance analysis for deletions for 25 Phase 31000 Genomes Project individuals with CNV calls and high coverage whole genome sequence. SNV: single nucleotide variant, TP: true positive, FN: false negative, TN: true negative, FP: false positive.

\begin{tabular}{|l|l|l|l|l|l|l|l|}
\hline $\begin{array}{l}\text { Number of } \\
\text { heterozygous SNVs }\end{array}$ & Total & TP & FN & TN & FP & Sensitivity & Specificity \\
\hline $1-2$ & 703 & 126 & 577 & 652 & 51 & 0.179232 & 0.927453 \\
\hline $3-9$ & 714 & 434 & 280 & 627 & 87 & 0.607843 & 0.878151 \\
\hline $10-19$ & 452 & 341 & 111 & 393 & 59 & 0.754425 & 0.869469 \\
\hline $20-49$ & 784 & 640 & 144 & 665 & 119 & 0.816327 & 0.848214 \\
\hline $50-99$ & 643 & 581 & 62 & 527 & 116 & 0.903577 & 0.819595 \\
\hline $100-199$ & 695 & 639 & 56 & 551 & 144 & 0.919424 & 0.792805 \\
\hline $200-499$ & 489 & 460 & 29 & 346 & 143 & 0.940695 & 0.707566 \\
\hline $500+$ & 82 & 69 & 13 & 45 & 37 & 0.841463 & 0.548780 \\
\hline All & 7940 & 3290 & 4650 & 3806 & 4134 & 0.414358 & 0.479345 \\
\hline
\end{tabular}

Table 2. RBV performance analysis for duplications for 25 Phase 31000 Genomes Project individuals with CNV calls and high coverage whole genome sequence. SNV: single nucleotide variant, TP: true positive, FN: false negative, TN: true negative, FP: false positive.
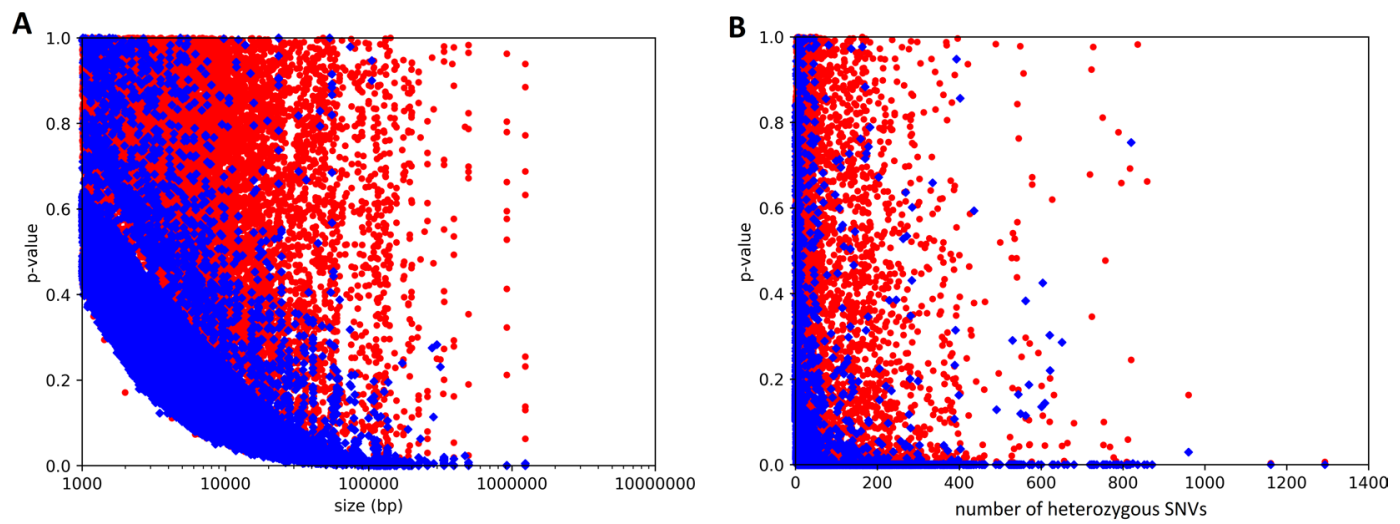

- Random diploid regions

Figure 3. Ability of RBV to prioritise authentic CNVs. Comparision between the results from $31,791 \mathrm{CNV}$ from 25 Phase 31000 Genomes Project individuals ${ }^{39}$ and randomly generated diploid regions with the same number of callalble positions as each deletion, or number of heterozygous positions for each duplication. (A) Performance of RBV for 23,851 deletions. (B) Performance of RBV for 7,940 duplications.

of $13.2 \%, 50-100 \mathrm{~kb}$ had a sensitivity of $84 \%$, and deletions $>100 \mathrm{~kb}$ had a sensitivity of $100 \%$. Full analyses are presented in the Supplementary Data.

Use cases. We established the ability of RBV to correctly identify CNVs in the context of causative mutations using two use cases. Firstly, our in-house CNV filtering and prioritisation pipeline (including RBV) was applied 
to WGS reads from two siblings who presented with recurrence of ataxia, deafness, developmental delay, rhabdomyolysis, cardiomyopathy and hypothyroidism ${ }^{33}$. The pipeline identified a $34 \mathrm{~kb}$ deletion encompassing exons three to nine (NC_000022.10:g.20028959_20062955del33997) resulting in nullizygosity over this region in both siblings. Using default parameters and the GATK callable intervals file, RBV validated the presence of this CNV in both siblings when compared to other regions in the genome of the same size resulting in $p$-values of $p=0.043$ and $\mathrm{p}=0.027$, with no heterozygous SNVs present across the deleted region for both siblings.

Another use for RBV is to test the potential inheritance of CNVs. Using HTS our laboratory recently identified a causative heterozygous 19.6 Mb 2q37 terminal deletion (GRCh37 Chr2:233834098-253404903; NC_000002.11:g. 233834098_253404903del) in a child with ASD ${ }^{34}$. There were both WES and WGS data available for the affected child, and WES data for the parents. RBV was run with default parameters and the GATK callable intervals file, for all four sequence sources. RBV confirmed the deletion with $\mathrm{p}$-value $=0.0$ from variants called from both WES and WGS from the affected child (with 0 and 92 heterozygous SNVs out of a total 2,930 and 58,396 variants called in the vcf file over the region, respectively). In comparison, the two parents had 284 and 294 heterozygous SNVs in the exonic sequence in the same region (out of 3,089 and 3,154 total variants called), resulting in p-values of 0.898 and 0.936 , respectively. Thus RBV provided evidence that the causative deletion was absent in the parents and is therefore de novo, confirmed by Sanger sequencing.

\section{Discussion}

As more research and diagnostic centres investigate the identification of CNVs through sequence data, there is increasing need for the ability to prioritise clinically relevant variants called from CNV detection software platforms. Although a number of detection tools use read depth, split reads, read pairs, and assembly-based techniques, the utility of read balance in $\mathrm{CNV}$ analysis has so far been largely underutilised. Thus, RBV was developed to exploit this additional piece of sequence information to reinforce calls from $\mathrm{CNV}$ calling pipelines, allowing for prioritisation of variants in the identification of pathogenic CNVs when used in conjunction with functional annotations.

We compared the results of RBV from 31,791 CNVs and randomly generated diploid regions. From this we were able to display the ability of RBV to differentiate genuine deletions $>30 \mathrm{~kb}$ and duplications with $>20$ heterozygous SNVs from diploid loci. Thus, this software has utility in prioritising putatively pathogenic deletions $>30 \mathrm{~kb}$ and duplications with $>20$ heterozygous SNVs. However, the sensitivity and specificity of RBV decreases for smaller variants with fewer heterozygous SNVs.

One limitation of the analyses performed by RBV results from the tendency of CNV breakpoints to occur as a result of replication errors within fragile sites or other repetitive elements ${ }^{35-37}$. Due to the low sequence complexity of such elements, these regions can be problematic for alignment and variant calling algorithms, resulting in low confidence SNV calls which are often excluded from callable intervals files. As such, if a callable intervals file is included, the search space for CNVs will be reduced, decreasing the ability of the software to sensitively identify true CNVs. Without the inclusion of a callable intervals file, variant callers will have a reduced accuracy in calling SNVs, which will subsequently result in a decreased ability of RBV to sensitively identify true duplications and potentially decrease the specificity for deletions. Thus, the propensity for CNV breakpoints to occur within repetitive regions is potentially partially responsible for RBVs performance bias for larger CNVs.

We were also able to demonstrate the execution of RBV using three clinical cases (two families), including successful identification of a $34 \mathrm{~kb}$ causative deletion from WGS, and the identification of a $19.6 \mathrm{Mb}$ deletion from WGS and WES, along with confirmation of mode of inheritance.

\section{Conclusions}

$\mathrm{RBV}$ is a software tool designed to assist in the rapidly expanding speciality of identifying clinically relevant CNVs through prioritisation.

The software includes utility for both multiplication and deletion analysis of nominated CNV sites from both WES and WGS data. Sample data for the operation of RBV is available via the GitHub repository.

Ethics approval and consent to participate. The 1000 Genomes Project Phase 3 data was obtained directly from the The International Genome Sample Resource made available under the Fort Lauderdale Agreement $^{38}$.

The genetic analysis and de-identified publication of variants for use cases was performed under the approval of the New Zealand Northern B Health and Disability Ethics Committee (12/NTB/59) in accordance with guidelines and regulations in the Ethical Guidelines for Observational Studies from the New Zealand National Ethics Advisory Committee. Parents provided written informed consent.

Availability and requirements. Project name: RBV. Project home page: https://github.com/whitneywhitford/RBV. Operating system(s): Linux.Programming language: Python 2.7. Other requirements: SAMtools 1.3 or higher, tabix. License: GPL v3. Any restrictions to use by non-academics: None.

\section{Data availability}

The datasets analysed for the performance of RBV are available via request.

Received: 17 July 2018; Accepted: 25 October 2019;

Published online: 15 November 2019

\section{References}

1. Feuk, L., Carson, A. R. \& Scherer, S. W. Structural variation in the human genome. Nat. Rev. Genet. 7, 85-97 (2006)

2. Kruglyak, L. \& Nickerson, D. A. Variation is the spice of life. Nat. Genet. 27, 234-236 (2001).

3. Conrad, D. F. et al. Origins and functional impact of copy number variation in the human genome. Nature 464, 704-712 (2010). 
4. Durbin, R. M. et al. A map of human genome variation from population-scale sequencing. Nature 467, 1061-1073 (2010).

5. McVean, G. A. et al. An integrated map of genetic variation from 1,092 human genomes. Nature 491, 56-65 (2012).

6. Pang, A. W. et al. Towards a comprehensive structural variation map of an individual human genome. Genome Biol. 11, R52 (2010).

7. Stranger, B. E. et al. Relative Impact of Nucleotide and Copy Number Variation on Gene Expression Phenotypes. Science (80-.). 315, 848-853 (2007).

8. Sellier, C. et al. Decreased DGCR8 expression and miRNA dysregulation in individuals with 22q11.2 deletion syndrome. PLoS One 9, e103884 (2014).

9. Whitford, W. et al. Compound heterozygous SLC19A3 mutations further refine the critical promoter region for biotin-thiamineresponsive basal ganglia disease 3 (2017).

10. Finelli, P. et al. Juxtaposition of heterochromatic and euchromatic regions by chromosomal translocation mediates a heterochromatic long-range position effect associated with a severe neurological phenotype. Mol. Cytogenet. 5, 16 (2012).

11. Malhotra, D. \& Sebat, J. CNVs: Harbingers of a rare variant revolution in psychiatric genetics. Cell 148, 1223-1241 (2012).

12. Miller, D. T. et al. Consensus Statement: Chromosomal Microarray Is a First-Tier Clinical Diagnostic Test for Individuals with Developmental Disabilities or Congenital Anomalies. Am. J. Hum. Genet. 86, 749-764 (2010).

13. Liang, D. et al. Copy number variation sequencing for comprehensive diagnosis of chromosome disease syndromes. J. Mol. Diagnostics 16, 519-526 (2014).

14. OMICtools. Whole-genome sequencing software tools. OMIC tools Available at, http://omictools.com/whole-genomeresequencing-category (Accessed: 7th March 2016) (2016).

15. Zhao, M., Wang, Q. Q., Wang, Q. Q., Jia, P. \& Zhao, Z. Computational tools for copy number variation (CNV) detection using nextgeneration sequencing data: features and perspectives - Springer. BMC Bioinformatics 14(Suppl 1), S1 (2013).

16. Chen, H., Bell, J. M., Zavala, N. A., Ji, H. P. \& Zhang, N. R. Allele-specific copy number profiling by next-generation DNA sequencing. Nucleic Acids Res. 43, 1-14 (2015).

17. Favero, F. et al. Sequenza: Allele-specific copy number and mutation profiles from tumor sequencing data. Ann. Oncol. 26, 64-70 (2015).

18. Holt, C. et al. WaveCNV: Allele-specific copy number alterations in primary tumors and xenograft models from next-generation sequencing. Bioinformatics 30, 768-774 (2014).

19. Lai, Y. Change-Point Analysis of Paired Allele-Specific Copy Number Variation Data. J. Comput. Biol. 19, 679-693 (2012).

20. Li, Y., Zhou, S., Schwartz, D. C. \& Ma, J. Allele-Specific Quantification of Structural Variations in Cancer Genomes. Cell Syst. 3, 21-34 (2016)

21. Van Loo, P. et al. Allele-specific copy number analysis of tumors. Proc. Natl. Acad. Sci. 107, 16910-16915 (2010).

22. Marenne, G., Chanock, S. J., Malats, N. \& Génin, E. Advantage of Using Allele-Specific Copy Numbers When Testing for Association in Regions with Common Copy Number Variants. PLoS One 8, 1-12 (2013).

23. Mayrhofer, M., DiLorenzo, S. \& Isaksson, A. Patchwork: allele-specific copy number analysis of whole-genome sequenced tumor tissue. Genome Biol. 14, R24 (2013).

24. Zhang, Z. \& Hao, K. SAAS-CNV: A Joint Segmentation Approach on Aggregated and Allele Specific Signals for the Identification of Somatic Copy Number Alterations with Next-Generation Sequencing Data. PLoS Comput. Biol. 11, 1-27 (2015).

25. Wang, W., Wang, W., Sun, W., Crowley, J. J. \& Szatkiewicz, J. P. Allele-specific copy-number discovery from whole-genome and whole-exome sequencing. Nucleic Acids Res. 43, 1-18 (2015).

26. Zhu, M. et al. Using ERDS to infer copy-number variants in high-coverage genomes. Am. J. Hum. Genet. 91, 408-21 (2012).

27. DePristo, M. A. et al. A framework for variation discovery and genotyping using next-generation DNA sequencing data. Nat. Genet. 43, 491-498 (2011)

28. Li, H. et al. The Sequence Alignment/Map format and SAMtools. Bioinformatics 25, 2078-2079 (2009).

29. Garrison, E. \& Marth, G. Haplotype-based variant detection from short-read sequencing (2012).

30. Rimmer, A. et al. Integrating mapping-, assembly- and haplotype-based approaches for calling variants in clinical sequencing applications. Nat. Genet. 46, 912-8 (2014).

31. Li, H. \& Durbin, R. Fast and accurate short read alignment with Burrows-Wheeler transform. Bioinformatics 25, 1754-1760 (2009).

32. The 1000 Genomes Project Consortium. A global reference for human genetic variation. Nature 526, 68-74 (2015).

33. Whitford, W. Identification of Genetic Copy Number Variants in Neurodevelopmental Disorders from Genome Sequence Data. (The University of Auckland, 2019).

34. Swan, B. W. Exome Sequencing Applied To Discover Variants in Autism Spectrum Disorder and Neurodevelopmental Conditions. (University of Auckland, 2017).

35. Wei, P.-C. et al. Long Neural Genes Harbor Recurrent DNA Break Clusters in Neural Stem/Progenitor Cells. Cell 164, 644-655 (2016).

36. Wilson, T. E. et al. Large transcription units unify copy number variants and common fragile sites arising under replication stress. Genome Res. 25, 189-200 (2015).

37. Cardoso, A. R., Oliveira, M., Amorim, A. \& Azevedo, L. Major influence of repetitive elements on disease-associated copy number variants (CNVs). Hum. Genomics 10, 30 (2016).

38. Wellcome Trust. Sharing Data from Large-scale Biological Research Projects: A System of Tripartite Responsibility (2003).

39. Sudmant, P. H. et al. An integrated map of structural variation in 2,504 human genomes. Nature 526, 75-81 (2015).

\section{Acknowledgements}

The authors wish to acknowledge the contribution of NeSI high-performance computing facilities to the results of this research. NZ's national facilities are provided by the NZ eScience Infrastructure and funded jointly by NeSI's collaborator institutions and through the Ministry of Business, Innovation \& Employment's Research Infrastructure programme. URL https://www.nesi.org.nz. We thank Malcolm de Raat for coding guidance. J.C.J. was supported by a Rutherford Discovery Fellowship from the New Zealand government, administered by the Royal Society of New Zealand. The research was funded by the Minds for Minds Charitable Trust, the IHC Foundation and the Oakley Mental Health Foundation.

\section{Author contributions}

All authors were involved in the concept design and refinement of RBV. W.W. developed and tested the software, and wrote the manuscript. J.C.J. critically reviewed the manuscript. All authors read and approved the final manuscript.

\section{Competing interests}

The authors declare no competing interests. 


\section{Additional information}

Supplementary information is available for this paper at https://doi.org/10.1038/s41598-019-53181-7.

Correspondence and requests for materials should be addressed to W.W.

Reprints and permissions information is available at www.nature.com/reprints.

Publisher's note Springer Nature remains neutral with regard to jurisdictional claims in published maps and institutional affiliations.

(c) (i) Open Access This article is licensed under a Creative Commons Attribution 4.0 International License, which permits use, sharing, adaptation, distribution and reproduction in any medium or format, as long as you give appropriate credit to the original author(s) and the source, provide a link to the Creative Commons license, and indicate if changes were made. The images or other third party material in this article are included in the article's Creative Commons license, unless indicated otherwise in a credit line to the material. If material is not included in the article's Creative Commons license and your intended use is not permitted by statutory regulation or exceeds the permitted use, you will need to obtain permission directly from the copyright holder. To view a copy of this license, visit http://creativecommons.org/licenses/by/4.0/.

(c) The Author(s) 2019 